\title{
SÍNTESE E AVALIAÇÃO DE AZODERIVADO DE 2-FENILBENZOXAZOL COMO SUBSTRATO FLUOROGÊNICO NA DIFERENCIAÇÃO DE Candida spp.
}

\author{
SOUZA, P.B. ${ }^{1 ;}$ CORBELLINI, V.A. ${ }^{2}$
}

PALAVRAS-CHAVE: 2-fenilbenzoxazol, Candida spp., biotransformação.

\begin{abstract}
RESUMO
Neste estudo foram sintetizados três derivados de 2-fenilbenzoxazol (2FBO) funcionalizados com a função azo: (2'-hidroxi-5'-fenilazofenil)benzoxazol (HFAZBO), 2-(2'-hidroxi-5'-(4"-carboxi)fenilazofenil)benzoxazol (HFAZBO$\left.\mathrm{CO}_{2} \mathrm{H}\right)$ e 2-(2'-hidroxi-5'-(4"-nitro)fenilazofenil)benzoxazol (HFAZBO-NO 2 ). Os compostos foram utilizados como substratos fluorogênicos para agentes de infecção urinária, representantes de Candida spp., para explorar suas características metabólicas. A caracterização estrutural dos compostos foi realizada por meio de Espectroscopia de Absorção Molecular no Infravermelho com transformada de Fourier (FT-IR) e por determinação da faixa de fusão. Os ensaios fluorogênicos foram realizados em espécies de Candida spp.: Candida albicans ATCC 10231, Candida famata RL38, Candida glabrata 06130L, Candida glabrata RL43, Candida glabrata 0030L, Candida guilliermondii TH07, Candida krusei ATCC 6258, Candida krusei 0037L, Candida parapsilosis (RL33), Candida parapsilosis (RL38), Candida stellatoidea (9875), Candida stellatoidea (3613), Candida tropicalis ATCC 750. Não foi observada alteração sob luz UV nas leveduras testadas. Do mesmo modo, o extrato obtido a partir das colônias não apresentou fluorescência sob luz UV. Dessa forma, faz-se necessário desenvolver novos estudos que avaliem a função metabólica de Candida spp. a partir da biotransformação levando a liberação de moléculas fluorescentes.
\end{abstract}

\section{SYNTHESIS AND EVALUATION OF AZODERIVATED FROM 2-PHENYLBENZOXAZOLE AS A FLUOROGENIC SUBSTRATE FROM Candida SPP. DIFERENCIATION}

KEY-WORDS: 2-phenylbenzoxazole, Candida spp., biotransformation.

\begin{abstract}
In this study, three 2-phenylbenzoxazole derivatives (2FBO) functionalized with an azo function: (2'-hydroxy-5'phenylazophenyl) benzoxazole (HFAZBO), 2- (2'-hydroxy-5'-(4" -carboxy) phenylazophenyl) benzoxazole (HFAZBO$\mathrm{CO} 2 \mathrm{H})$ and 2-(2'-hidroxy-5'-(4"-nitro)phenylazophenyl)benzoxazole $\left(\mathrm{HFAZBO}^{-} \mathrm{NO}_{2}\right)$. The substrates were used as fluorogenic substrates for urinary infection agents, Candida spp. representatives, to facilitate their metabolic characteristics. The molecular characterization of the compounds was performed by Fourier Transform Infrared Molecular Absorption Spectroscopy (FT-IR) and by determination of the melting range. Fluorogenic assays were performed on Candida spp. species: Candida albicans ATCC 10231, Candida famata RL38, Candida glabrata 06130L, Candida glabrata RL43, Candida glabrata 0030L, Candida guilliermondii TH07, Candida krusei ATCC 6258, Candida krusei 0037L, Candida parapsilosis (RL33 ), Candida parapsilosis (RL38), Candida stellatoidea (9875), Candida stellatoidea (3613), Candida tropicalis ATCC 750. No change was observed in UV light in yeast tested. Likewise, the extract was removed from the colonies without fluorescence under UV light. Thus, it is necessary to develop new studies that evaluate a metabolic function of Candida spp. from the biotransformation leading to a release of fluorescent molecules.
\end{abstract}

\footnotetext{
${ }^{1}$ Acadêmica do curso de Farmácia na Universidade de Santa Cruz do Sul.

2 Docente do Departamento de Química e Física na Universidade de Santa Cruz do Sul.
} 


\section{INTRODUÇÃO}

A partir de 1940 ensaios biológicos começaram a ser explorados para a diferenciação de microorganismos com base na metabolização de corantes. Os primeiros trabalhos foram realizados em cultura de células bacterianas crescendo sobre meio sólido, ao qual era incorporada a fenolftaleína (BRAY; KING, 1943).

Substratos cromogênicos são úteis na composição de meios de cultura para diferenciação de microorganismos tendo evoluído para a comercialização do ágar Rambach que possibilitou a diminuição no tempo de análise microbiológica. Esse tipo de substrato continua sendo explorado com capacidade diagnóstica para a detecção de enzimas (RAMBACH, 1990; CELLIER et al., 2011).

Derivados cumarínicos de 4-metilumbeliferona (4-MU) ou 7-amino-4-metilcumarina (7-AMC) passaram a ser frequentemente utilizados como substratos fluorogênicos com a proposta de detectar grupos específicos de enzimas em micro-organismos após visualização sob luz UV de $365 \mathrm{~nm}$ com potencial para diferenciação de patógenos (GRIST, 1987; MANAFI, 1991).

$\mathrm{Na}$ atualidade, substratos fluorogênicos de diferentes classes químicas são utilizados em estudos buscando a caracterização ou a especificidade enzimática de micro-organismos, sendo avaliados como ferramenta promissora para diagnósticos, uma vez que, contribuem para a elucidação do comportamento metabólico de cepas (HODGES et al., 2018; BASBOUS et al., 2018; ELDIPINA, 2019). Entre os substratos utilizados em ensaios fluorogênicos e cromogênicos destacam-se os compostos azóicos (BRIGHENTI et al., 2014).

Esses corantes são caracterizados pela presença de ligação diazo e apresentam uma ampla gama de aplicações em diversos campos (IANDOLO et al., 2011;REZAEI-SERESHT et al., 2017). Compostos azóicos ligados a benzoxazolas têm seu potencial de fluorescência aumentado, em especial quando o produto é obtido na presença de $\mathrm{Zn} \mathrm{2+,} \mathrm{em} \mathrm{decorrência} \mathrm{do} \mathrm{processo} \mathrm{de} \mathrm{inibição} \mathrm{da} \mathrm{transferência} \mathrm{protônica} \mathrm{intramolecular} \mathrm{no}$ estado excitado (SHANG et al., 2018).

Os corantes com grupamento azo podem ser biotransformados por microorganismos que possuem a enzima azorredutase como Enterococcus faecalis, Klebsiella pneumoniae, Pseudomonas aeruginosa NBAR 12, Bacillus cereus e Aspergillus flavus, individualmente ou em consórcio microbiano. Essa característica tem sido explorada no contexto ambiental, como forma de remoção desses contaminantes provenientes de efluentes industriais (BHATT et al. 2005; ESMAELI; KALANTARI, 2016; KANAGARAJ et al., 2012; DIXIT; GARG, 2018; ESLAMI et al., 2019).

Entre os micro-organismos que possuem a enzima azorredutase, encontra-se o gênero Candida, que possui mais de vinte espécies consideradas patógenos comensais com características oportunistas sendo capazes de causar doenças em indivíduos imunossuprimidos como os portadores do HIV, pacientes que sofreram queimaduras graves e pacientes com diabetes não controlado que está associado a aumento na resistência dos micro-organismos ao tratamento (DEIVASIGAMANI; DAS, 2011; HERNÁNDEZ-SOLÍs; RUEDA-GORDILLO; ROJASHERRERA, 2014; RAMOS et al., 2016; MALMARTEL; GHASAROSSIAN, 2016).

Espécies emergentes de Candida spp. tornaram-se importantes patógenos cosmopolitas em especial no contexto hospitalar, sendo capazes de driblar os mecanismos de ação de fármacos (OSTROSKY-ZEICHNER, 2013; CLANCY; NGUYENM, 2016). As maiores taxas de isolamento de Candida podem ser encontradas em indivíduos com idade superior a 60 anos. Em relação ao gênero, é reportada maior ocorrência de candidúria em mulheres do que em homens (OZER; DURMAZ; YULA, 2016; ORTIZ et al., 2018). Para contribuir no diagnóstico 
microbiológico, podem ser citados os derivados de 2-fenilbenzoxazol para serem utilizados em ensaios fluorogênicos dada sua alta capacidade de emissão de fluorescência (HOLLER et al., 2002).

Do ponto de vista da bioinformática, a caracterização metabólica de compostos ou micro-organismos pode ser realizada pela associação de quimiometria e Espectroscopia de Absorção Molecular no Infravermelho com transformada de Fourier (FT-IR) a qual apresenta vantagens como alta sensibilidade e pouca utilização de reagentes (AMIALI et al., 2011). Devido às suas propriedades, a FT-IR constitui uma ferramenta atual que vem sendo utilizada na verificação de metabolização de substâncias presentes no meio de crescimento pelos microorganismos ou ainda, na investigação de parâmetros eletroquímicos desse tipo de amostra (YOU et al., 2019; ULDIS; MUTER, 2019).

Neste contexto, foi avaliada a síntese de novos derivados azóicos para verificar o seu potencial para diferenciação de espécies de Candida app. através da sua ação como substratos fluorogênicos.

\section{MATERIAIS E MÉTODOS}

\subsection{Síntese e caracterização físico-química dos derivados de 2-fenilbenzoxazol (2-PBO)}

$\mathrm{Na}$ rota escolhida para a síntese dos compostos, conforme Figura 1, foi constituído, inicialmente, o núcleo benzoxazólico por condensação de ácido salicílico, previamente dissolvido em NaOH equimolar, com 2aminofenol (2AF) em ácido polifosfórico (PPA) seguido da diazotação com anilina, ácido 4-aminobenzoico (PABA) ou p-nitroanilina. Todos os reagentes e solventes utilizados foram de grau analítico. Os pontos de fusão foram medidos em fusiômetro (Quimis ${ }^{\circledR}$ ). Para isso, alíquotas das amostras secas foram inseridas no capilar do equipamento e submetidas a aquecimento com temperatura controlada. Os espectros foram obtidos pela técnica de Espectroscopia de Infravermelho com transformada de Fourier em espectrômetro (Spectrum 400 FT-IR Perkin Elmer ${ }^{\circledR}$ ) com acessório de Reflectância Total Atenuada (ATR) na faixa de 4000 a $600 \mathrm{~cm}^{-1}, 4 \mathrm{~cm}^{-1} \mathrm{de}^{-}$ resolução e 8 scans.

Figura 1: Esquema de síntese dos derivados de 2-PBO<smiles>O=C(O)c1ccccc1O</smiles>

[1]<smiles>Nc1ccccc1O</smiles>

[2]

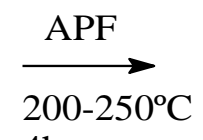

$4 \mathrm{~h}$<smiles>Oc1ccccc1-c1nc2ccccc2o1</smiles>

[3]

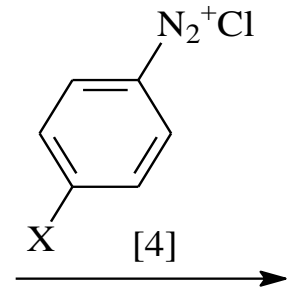

$0-5^{\circ} \mathrm{C}$

[4a] $\mathrm{X}=\mathrm{H}$

[4b] $\mathrm{X}=\mathrm{CO}_{2} \mathrm{H}$

[4c] $\mathrm{X}=\mathrm{NO}_{2}$

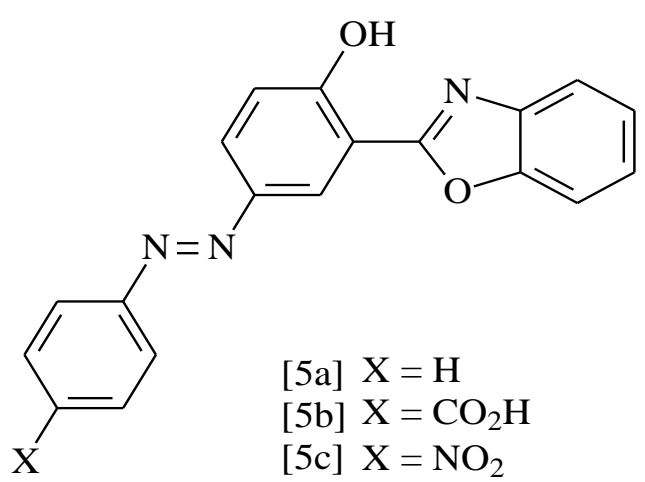

Revista Jovens Pesquisadores, Santa Cruz do Sul, v.9, n. 1, p. 47-58, jan./jun. 2019. https://online.unisc.br/seer/index.php/jovenspesquisadores/ 
2.1.1 2-(2'-hidroxi-5'-fenilazofenil)benzoxazol (HFAZBO-[5a]): Composto preparado a partir de 2-(2'hidroxifenilazofenil)benzoxazol (HBO) (0,211 g), NaOH (40 g.t-1) (2 mL) e EtOH 96 \% (5 mL). Em banho de gelo com agitação magnética. Posteriormente, foi adicionada solução de anilina $(0,093 \mathrm{~g})$ em $\mathrm{HCl}(0,2 \mathrm{~mL})$ e $\mathrm{H}_{2} \mathrm{O}(0,2$ $\mathrm{mL})$, seguida da adição lenta de nitrito de sódio (5 mol.L-1) $(0,2 \mathrm{~mL})$. Rendimento: 61,06\%.Faixa de Fusão: 121$132{ }^{\circ}$ C. Fórmula molecular: $\mathrm{C}_{17} \mathrm{H}_{12} \mathrm{~N}_{3} \mathrm{O}_{2}(290$ g.mol-1).

2.1.2 2-(2'-hidroxi-5'-(4"'-carboxi)fenilazofenil)benzoxazol (HFAZBO- $\mathrm{CO}_{2} \mathrm{H}$, [5b]): Composto preparado a partir de 2(2'-hidroxifenilazofenil)benzoxazol (HBO) (0,633 g), NaOH (40 g.t-1) (6 mL) e EtOH 96 \% (15 mL). Em banho de gelo com agitação magnética. Posteriormente, foi adicionada solução de ácido-4-aminobenzoico $(0,411$ g) em $\mathrm{HCl}(0,6 \mathrm{~mL})$ e $\mathrm{H}_{2} \mathrm{O}(0,6 \mathrm{~mL})$, seguida da adição lenta de nitrito de sódio $\left(5 \mathrm{~mol} . \mathrm{L}^{-1}\right)(0,6 \mathrm{~mL})$. Rendimento: $78,07 \%$. Faixa de Fusão: $158-172^{\circ} \mathrm{C}$. Fórmula molecular: $\mathrm{C}_{18} \mathrm{H}_{13} \mathrm{~N}_{3} \mathrm{O}_{4}\left(335\right.$ g.mol$\left.{ }^{-1}\right)$.

2.1.3 2-(2'-hidroxi-5'-(4"'-nitro)fenilazofenil)benzoxazol (HFAZBO-NO2, [5c]): Composto preparado a partir de 2-(2'hidroxifenilazofenil)benzoxazol (HBO) (0,211g), NaOH (40 g.t-1) (2 mL) e EtOH 96 \% (5 mL). Em banho de gelo com agitação magnética. Posteriormente, foi adicionada solução de p-nitroanilina $(0,138 \mathrm{~g}) \mathrm{em} \mathrm{HCl}(0,2 \mathrm{~mL})$ e $\mathrm{H}_{2} \mathrm{O}(0,2 \mathrm{~mL})$, seguida da adição lenta de nitrito de sódio (5 mol.L-1) $(0,2 \mathrm{~mL})$. Rendimento: $65 \%$. Faixa de Fusão: 132-141 ${ }^{\circ}$ C. Fórmula molecular: $\mathrm{C}_{17} \mathrm{H}_{12} \mathrm{~N}_{4} \mathrm{O}_{4}$ (336 g.mol-1).

Os compostos sintetizados foram purificados de forma semelhante, por meio de recristalização. Para isso, foram utilizados de 15 a $20 \mathrm{~mL}$ de água a quente.

\subsection{Ensaios fluorogênicos}

Foram utilizadas cepas padrão de referência e isolados clínicos provenientes da Coleção de Microorganismos de Microbiologia Industrial da Universidade de Santa Cruz do Sul. As 14 cepas foram constituídas por Candida albicans ATCC 10231, Candida famata RL38, Candida glabrata 06130L, Candida glabrata RL43, Candida glabrata 0030L, Candida guilliermondii TH07, Candida krusei ATCC 6258, Candida krusei 0037L, Candida parapsilosis (RL33), Candida parapsilosis (RL38), Candida stellatoidea (9875), Candida stellatoidea (3613), Candida tropicalis ATCC 750. As cepas foram cultivadas em ágar Sabouraud. Em ensaio preliminar, foi utilizado o composto HFAZBO- $\mathrm{CO}_{2} \mathrm{H}$ como substrato fluorogênico, que foi adicionado de $0,1 \mathrm{~mL}$ de solução do composto (0,05mmol em dimetilsulfóxido-DMSO). As leveduras foram inoculadas em placas com $10 \mathrm{~mL}$ de ágar nutriente e glicose $1 \%$ tendo como controle negativo o mesmo meio de cultura sem a presença do SF. Como indicado na Figura 2, as placas foram incubadas a $35-37^{\circ} \mathrm{C}$ por 48 horas e visualizadas sob lâmpada UV de 360 $\mathrm{nm}$. Posteriormente, foi realizado ensaio confirmatório em que as amostras foram inoculadas, em duplicata, em tubos contendo $2 \mathrm{~mL}$ de caldo Sabouraud e incubadas a $35-37^{\circ} \mathrm{C}$ por $24 \mathrm{~h}$. Em seguida, foi adicionada alíquota de $50 \mu \mathrm{L}$ de HFAZBO- $\mathrm{CO}_{2} \mathrm{H}$ diluído em DMSO. No dia seguinte, as amostras foram submetidas à extração com 2 $\mathrm{mL}$ de solução saturada de cloreto de sódio e $0,5 \mathrm{~mL}$ de acetato de etila. Os tubos foram homogeneizados em vórtex e monitorados até que apresentassem separação de fases. A seguir, o sobrenadante resultante foi aplicado em cromatoplaca e submetido à análise por cromatografia em camada delgada (CCD, sílica HF254Merck) juntamente com HFAZBO- $\mathrm{CO}_{2} \mathrm{H}$ e 2-(2'-hidroxi-5'-aminofenil)benzoxazol (HAMBO) utilizando mistura de hexano:acetato de etila, 4:1. 


\section{RESULTADOS E DISCUSSÃO}

\subsection{Caracterização estrutural dos derivados de 2-fenilbenzoxazol (2-PBO)}

3.1.1 HFAZBO: Bandas FT-IR/ATR ( $\mathrm{cm}^{-1}$ ) (Figura 2): 3061,50 deformação axial (C-H) aromático; 1946,59; 1631,97 deformação axial $(C=N)$; 1587,10 - 1487,16 ligação axial $(C=C)$ aromático; 1259,10 - 1246,11 deformação axial assimétrica (C-O-C) do benzoxazol e vibração axial de (C-O) do fenol; 797,91-758,34 deformação angular fora do plano (C-H) aromático;

Figura 2: Espectro FTIR/ATR do composto HFAZBO

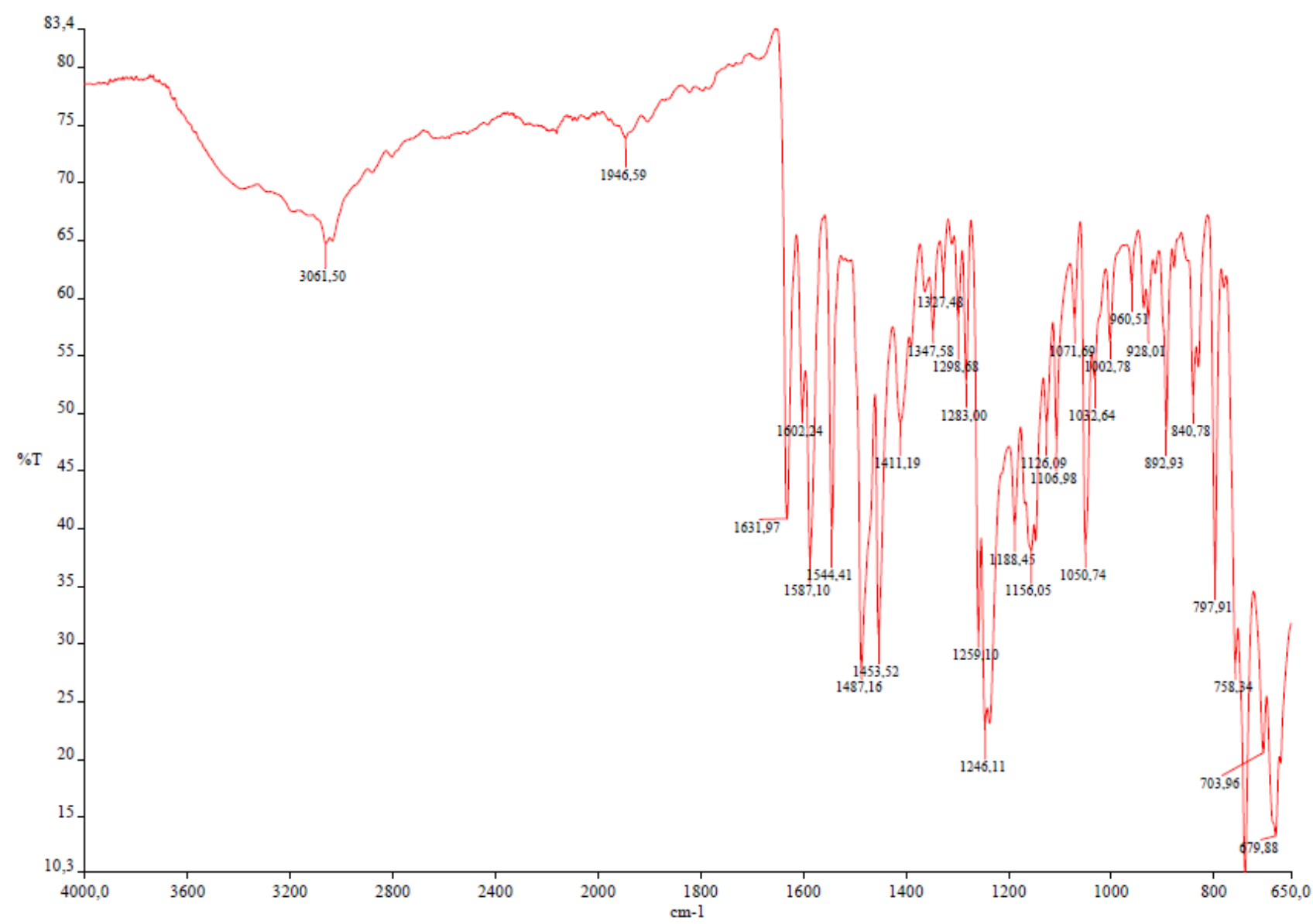

3.1.2 HFAZBO- $\mathrm{CO}_{2} \mathrm{H}$ : Bandas FT-IR/ATR ( $\mathrm{cm}^{-1}$ ) (Figura 3): 3339,45 deformação axial (-OH) associado; 1632,74 deformação axial de $(C=N)$ da benzoxazola; 1545,41-1489,31 ligação axial $(C=C)$ dos anéis aromáticos; 1260,691248,44 deformação axial assimétrica de (C-O-C) da benzoxazola; 740,04 deformação angular fora do plano de (C-H) aromático; 
Figura 3: Espectro FTIR/ATR do composto HFAZBO- $\mathrm{CO}_{2} \mathrm{H}$

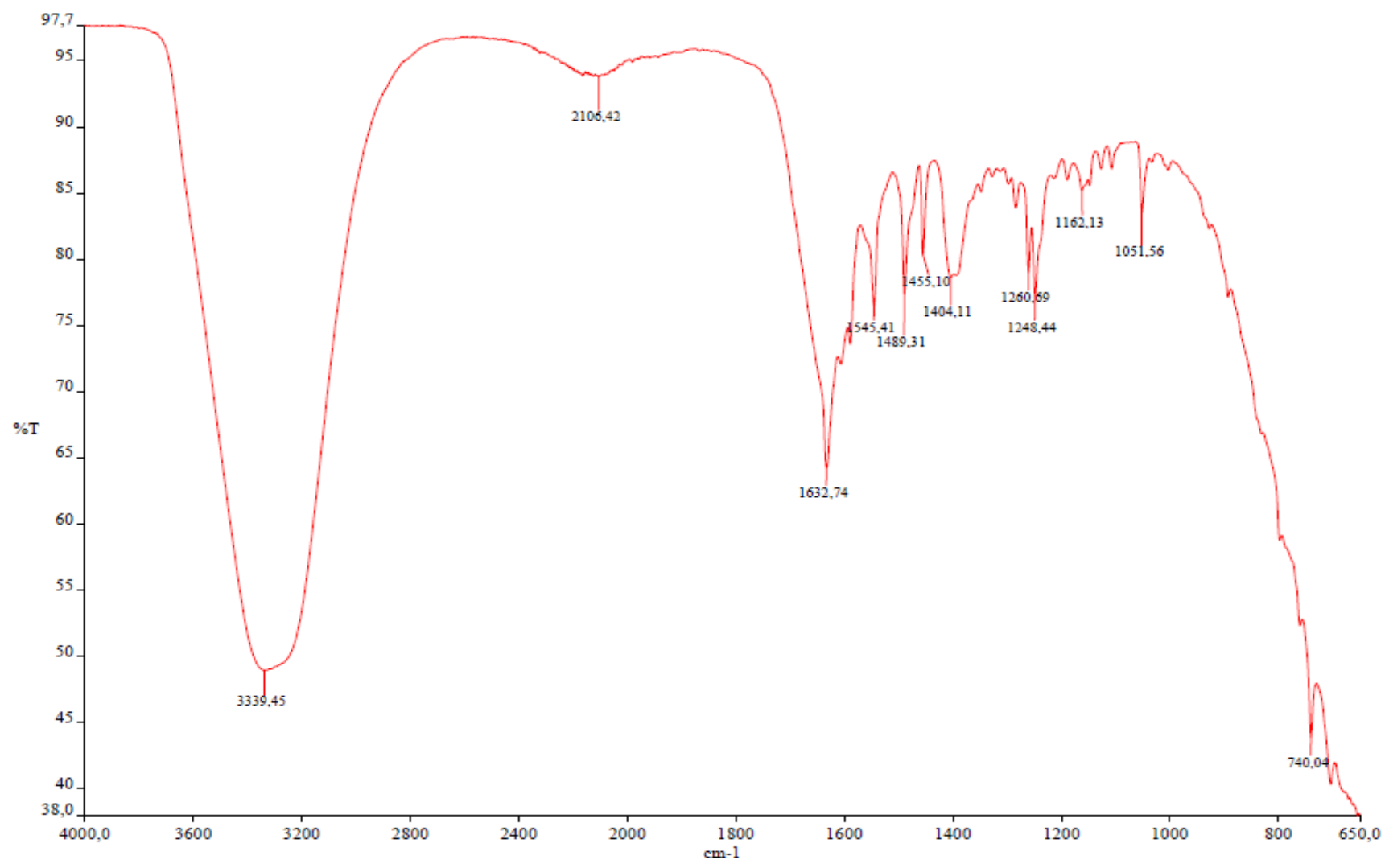

3.1.3 HFAZBO-NO 2 : Bandas FT-IR/ATR ( $\mathrm{cm}^{-1}$ ) (Figura 4): 1633,20 deformação axial de (C=N) da benzoxazola; 1588,20 - 1488,42 ligação axial de $(C=C)$ dos anéis aromáticos; 1545,42-1348,31 absorção assimétrica do grupo $\left(\mathrm{NO}_{2}\right)$; 1259,98-1248,01 deformação axial assimétrica de (C-0-C) da benzoxazola; 798,95 deformação angular fora do plano de (C-H) aromático.

Figura 4: Espectro FTIR/ATR do composto HFAZBO-NO 2

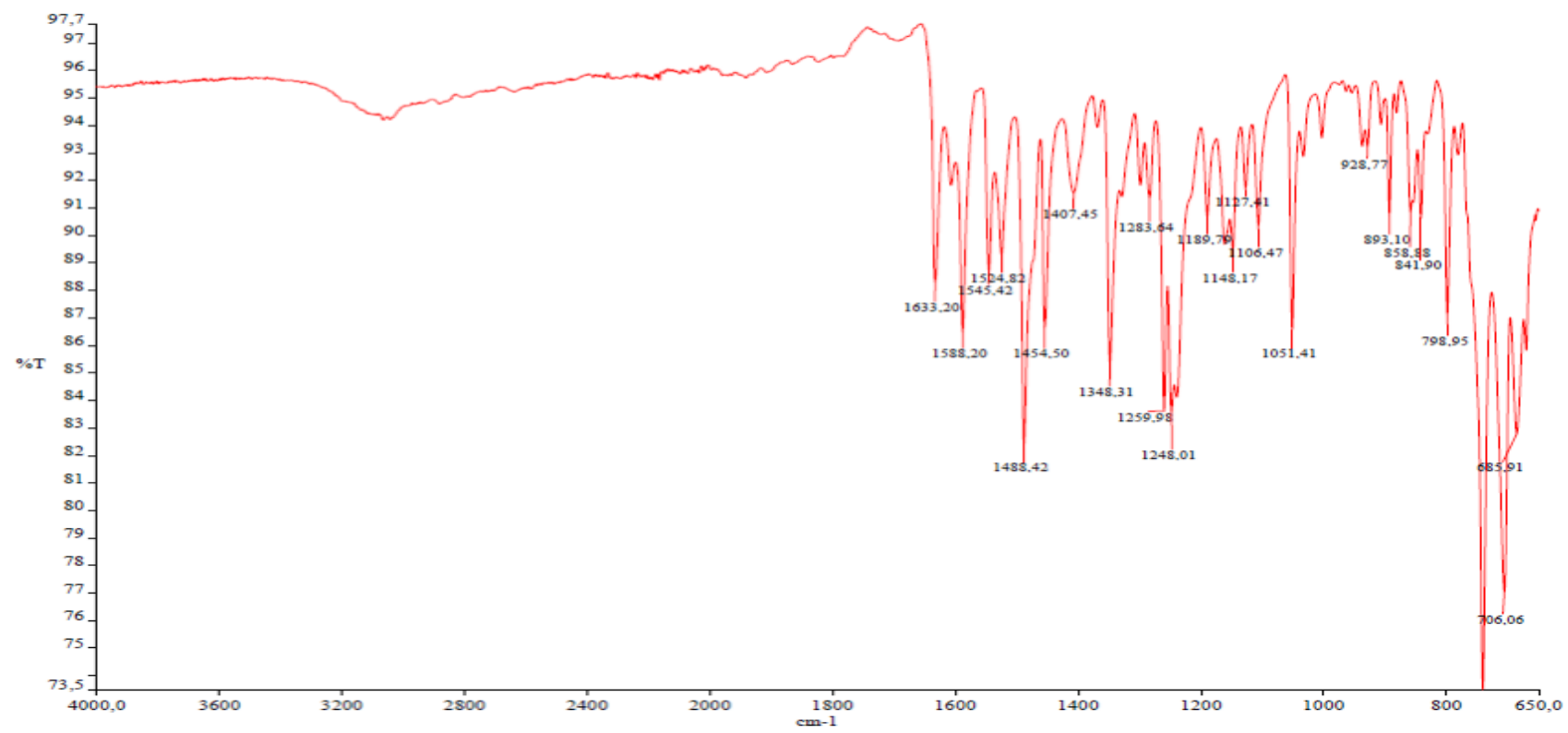


Os rendimentos obtidos na síntese dos derivados de 2-PBO são compatíveis com os percentuais disponíveis na literatura, que reporta a síntese de diversos derivados de N-(2-(3,4,5-trimetoxibenzil)-benzoxazol-5i) benzamida, em que foram obtidos rendimentos entre 49 a 70\% (KAUR et al., 2018). Por outro lado, podem ocorrer rendimentos inferiores, como na síntese de benzoxazolas dissubstituídas contendo grupamento sulfonil que levou a um rendimento de 35\% (ZEYREK et al., 2017). Podem ser utilizadas estratégias para aumento do rendimento como o uso de nano catalisadores, sendo possível obter 94\% do produto esperado (BHOSALE; BHANAGE, 2015).

A banda atribuída ao estiramento $\mathrm{C}=\mathrm{N}$ da benzoxazola é observada próxima a $1.626 \mathrm{~cm}^{-1}$, assim como em $1547 \mathrm{~cm}^{-1}$. Vibrações típicas da estrutura podem ser observadas em 1501 e $1489 \mathrm{~cm}^{-1}$. As bandas correspondentes ao estiramento assimétrico $\mathrm{Ar}-\mathrm{O} \_\mathrm{C}$ de benzoxazois pode ser observada em $1251 \mathrm{~cm}^{-1} \mathrm{ou} 1272$ $\mathrm{cm}^{-1}$. Na composição de polímeros, essas bandas são intensificadas quando há aumento do conteúdo de benzoxazois na estrutura (XUE-MIN DAI et al., 2019). Diferenças nas bandas para uma mesma estrutura podem ser explicadas pela possibilidade de modos vibracionais atribuídos a uma molécula de acordo com o total de graus de liberdade que ela possui (KELLNER et al, 2004).

Entre os comprimentos de onda $1725 \mathrm{~cm}^{-1}$ e $1700 \mathrm{~cm}^{-1}$, pode ser observada a banda correspondente ao ácido carboxílico, que está presente no composto HFAZBO- $\mathrm{CO}_{2} \mathrm{H}$ do mesmo modo, a banda correspondente à ligação C=0 deste grupamento pode ocorrer em $1661 \mathrm{~cm}^{-1}$ (ROEGES, 1994; NAKANISHI, 1962).

Os compostos que contêm grupo nitro são caracterizados pela presença de vibrações assimétricas e simétricas de estiramento de $\mathrm{NO}_{2}$ que ocorrem entre as regiões 1661 e $1499 \mathrm{~cm}^{-1}$ ou 1389 e $1259 \mathrm{~cm}^{-1}$, respectivamente (ROEGES, 1994; SILVERSTEIN; WEBSTER, 2006).

As bandas 1526, 1365 1351, $1201 \mathrm{~cm}^{-1}$ referentes a vibrações de alongamento; $556 \mathrm{~cm}^{-1}, 442 \mathrm{~cm}^{-1} \mathrm{de}^{-}$ deformação no plano e $702 \mathrm{~cm}^{-1}$ de deformação fora do plano são associadas ao grupamento nitro conforme a abordagem de cálculo de número de onda vibracional (BHAGYASREE, et al., 2014).

A porção da molécula constituída pelo benzeno apresenta vibrações a 1600, 1573 e $1524 \mathrm{~cm}^{-1}$ devido à ligação $\mathrm{C}=\mathrm{C}$ do anel. As vibrações de valência da ligação $\mathrm{C}-\mathrm{N}$ no grupo amino ligado ao anel benzeno são observadas a $1310 \mathrm{~cm}^{-1}$ para determinar a presença de anel aromático no espectro pode ser observada a presença de vibração de alongamento correspondente à ligação C-H que ocorre acima de $3000 \mathrm{~cm}^{-1} \mathrm{como}^{\mathrm{no}}$ intervalo de 3115 a $3065 \mathrm{~cm}^{-1}$ (COATES et al, 2000; MARY et al, 2012).

A ligação azo compondo complexos metálicos foi encontrada próxima a $1500 \mathrm{~cm}^{-1}$ (SARANGI; MAHAPATRA; SETHY, 2018). No entanto, alguns estudos demonstram que a ligação $\mathrm{N}=\mathrm{N}$ do grupo azo absorve próximo de $1429 \mathrm{~cm}^{-1}$, porém, não pode ser identificada em espectro de infravermelho, pois a natureza apolar da ligação proporciona bandas fracas. (LAMBERT et al., 1987; PAVIA; PAMPMAN; KRIZ, 1996; SILVERSTEIN, WEBSTER, 2006).

Embora haja dados na literatura que comprovem que espécies de Candida spp. possuem a enzima azorredutase (LUCAS et al., 2006; DEIVASIGAMANI; DAS, 2011), como observado nas Figuras 4, 5, 6, 7, 8 e 9, nos ensaios fluorogênicos realizados neste estudo não foi verificada biotransformação do substrato pelas cepas investigadas. Esse resultado pode ser explicado pela necessidade de ambiente microaerofílico para a atividade da enzima azorredutase, sendo que em micro-organismos aeróbicos, seu desempenho ideal é condicionado a um mediador redox (SHAH et al., 2013; ELFARASH et al., 2017). Deve ser levado em consideração que a enzima atua em pH e temperatura ideais e, a variável pH não foi controlada neste estudo. Em relação ao crescimento das cepas, não houve inibição pelo substrato utilizado. Tal resultado seria esperado, pois, estruturas com grupamento 
benzoxazólico apresentam potencial antifúngico inferior aos compostos benzotiazois, enquanto que, apresentam potencial de inibição moderada em cepas bacterianas (PADALKAR, 2016). Por outro lado, 2-benzoxazolas substituídas são vistas como potenciais fármacos antifúngicos, pois, podem apresentar mesma concentração inibitória mínima sobre crescimento de espécies de Candida spp. do que antifúngico padrão (RAMALINGAN et al. 2004). Do mesmo modo, não houve difusão do corante para o ágar, essencial para a diferenciação de espécies de micro-organismos que apresentam determinada atividade enzimática daqueles que não apresentam (CELLIER et al., 2014).

$\mathrm{Na}$ análise por CCD, foi desconsiderado o fator de retenção dos extratos, visto que todos percorreram a mesma distância sobre a placa cromatográfica. Bem como, não foi dado seguimento na investigação espectroscópica que levaria a possível via de degradação do corante (TAN, 2013).

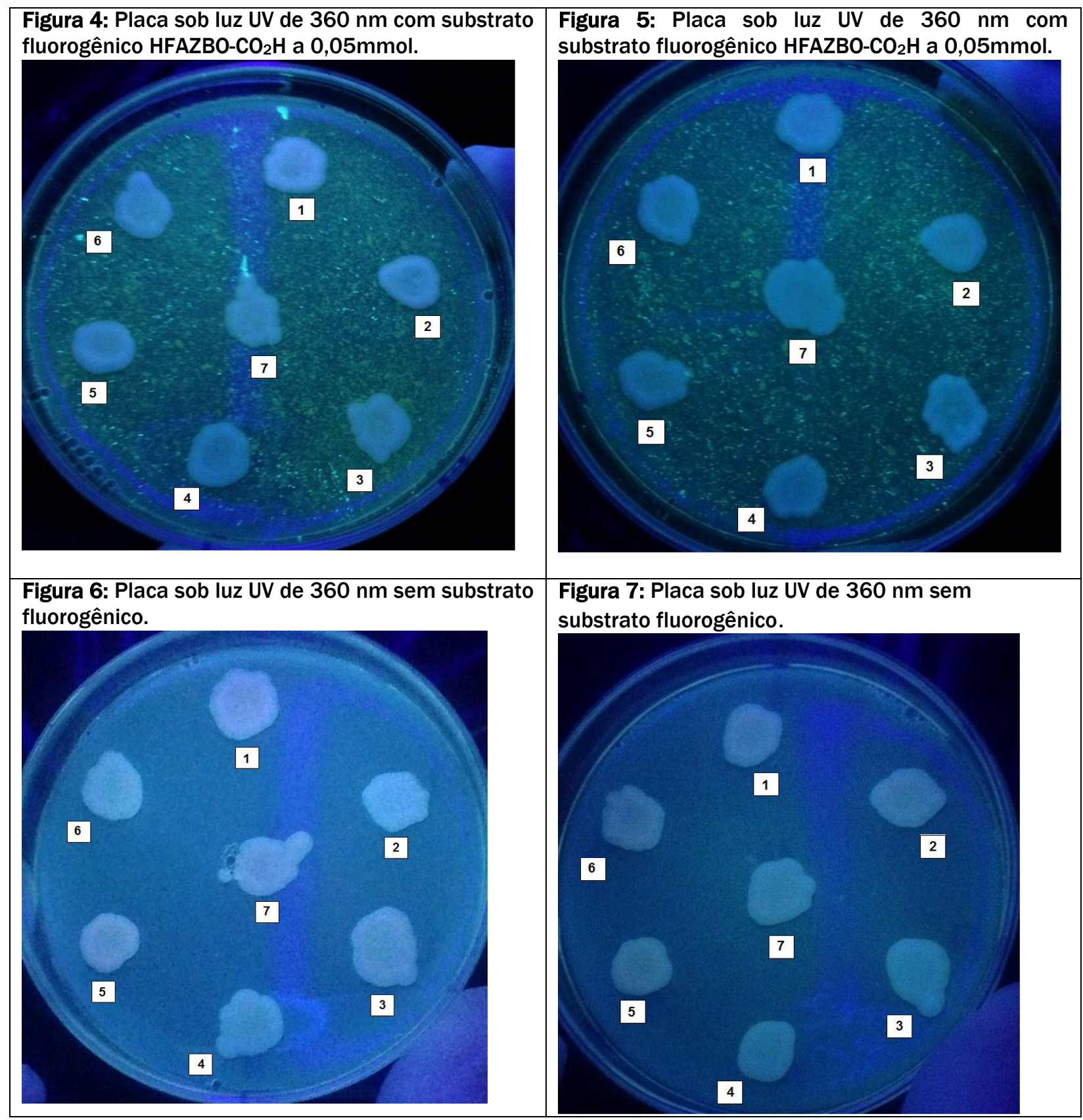

Revista Jovens Pesquisadores, Santa Cruz do Sul, v.9, n. 1, p. 47-58, jan./jun. 2019. https://online.unisc.br/seer/index.php/jovenspesquisadores/ 
Figura 8: Visualização da placa cromatográfica sob luz UV de $360 \mathrm{~nm}$. (1) HFAZBO- $\mathrm{CO}_{2} \mathrm{H}$, (2) HAMBO, (3) Candida albicans (ATCC 10231)/HFAZBO-CO $\mathrm{CO}_{2} \mathrm{H}$, (4) Candida albicans (ATCC 10231), (5) Candida famata (RL38)/HFAZBO$\mathrm{CO}_{2} \mathrm{H},(6)$ Candida famata (RL38), (7) Candida glabrata (06130L)/HFAZBO-CO ${ }_{2} \mathrm{H}$, (8) Candida glabrata (06130L), (9) Candida glabrata (RL49)/HFAZBO- $\mathrm{CO}_{2} \mathrm{H}$, (10) Candida glabrata (RL49), (11) Candida glabrata

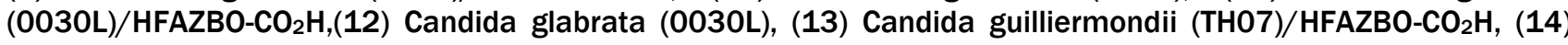
Candida guilliermondii (TH07), (15) Candida krusei (ATCC6258)/HFAZBO-CO $\mathrm{H}_{2}$ (16) Candida krusei (ATCC6258), (17) Candida krusei (0037L)/HFAZBO- $\mathrm{CO}_{2} \mathrm{H}$, (18) Candida krusei (0037L), (19) Candida krusei (ATCC6258)/HFAZBO-CO $2 \mathrm{H}$.

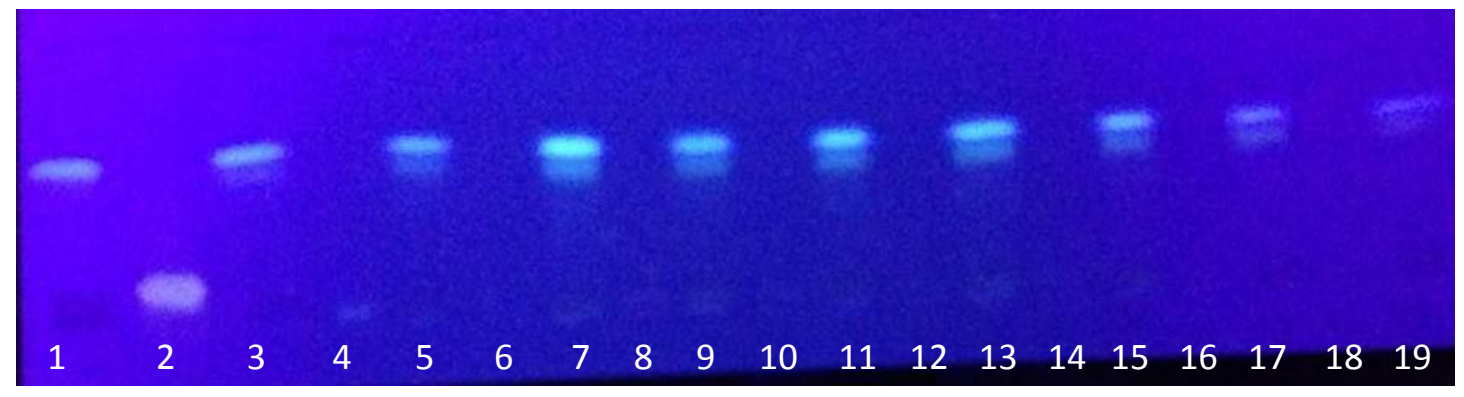

Figura 9: Visualização da placa cromatográfica sob luz UV de $360 \mathrm{~nm}$. (20) Candida krusei (ATCC6258),(21) Candida parapsilosis (RL33) / HFAZBO- $\mathrm{CO}_{2} \mathrm{H},(22)$ Candida parapsilosis (RL33), (23) Candida parapsilosis (RL38) /HFAZBO-CO ${ }_{2} \mathrm{H},(24)$ Candida parapsilosis (RL38), (25) Candida Stellatoidea (9875)/ $\mathrm{HFAZBO}-\mathrm{CO}_{2} \mathrm{H}$, (26) Candida Stellatoidea (9875), (27) Candida Stellatoidea (3613)/HFAZBO- $\mathrm{CO}_{2} \mathrm{H}$, (28) Candida Stellatoidea (3613), (29) Candida tropicalis (ATCC 750)/HFAZBO- $\mathrm{CO}_{2} \mathrm{H},(30)$ Candida tropicalis (ATCC 750).

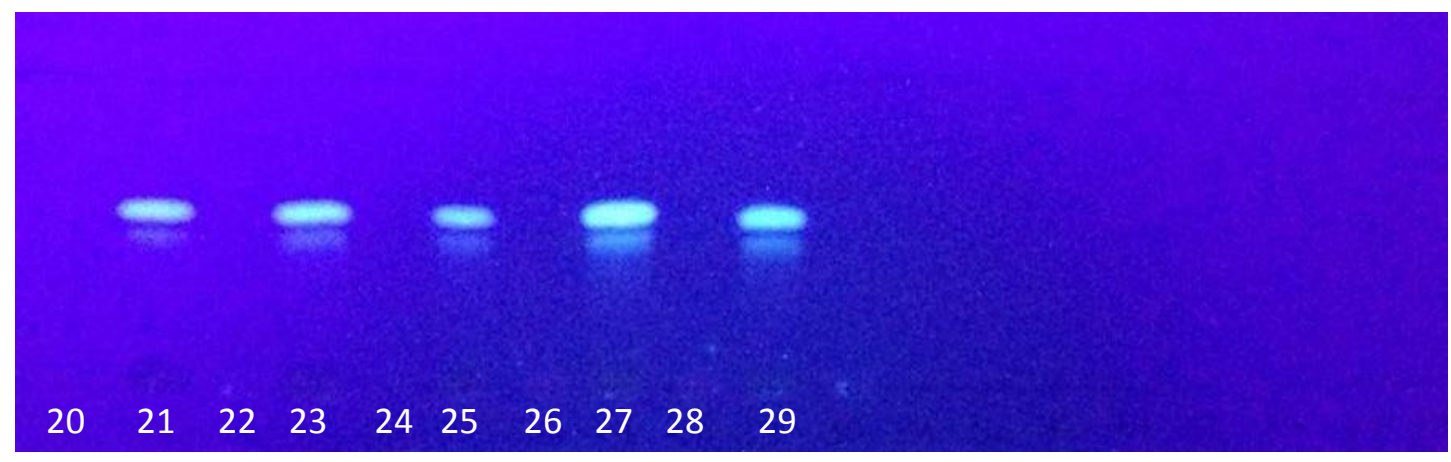

\section{CONSIDERAÇÕES FINAIS}

A síntese dos compostos esperados foi confirmada mediante FT-IR. No entanto, para otimização do procedimento de síntese e purificação, devem ser realizados novos experimentos, em replicata, e utilizando diferentes concentrações de substrato fluorogênico para os ensaios biológicos. A partir deste estudo, foi possível identificar uma concentração em que o substrato não é metabolizado pela levedura. Porém, novos estudos devem ser realizados para explorar a capacidade de biotransformação de corantes azóicos por espécies do gênero Cândida no contexto da metabolômica e sua utilidade para fins diagnósticos.

\section{REFERÊNCIAS}

AMIALI, Nassim M. et al. Rapid identification of community-associated methicillin-resistant Staphylococcus aureus by Fourier transform infrared spectroscopy. Diagnostic Microbiology and Infectious Disease. Vol. 70, n. 2, p. 157-166, 2011;

BASBOUS, Hind et al. Characterization of a glycyl-specific TET aminopeptidase complex from Pyrococcus horikoshii. Journal of Bacteriology. 2018 <https://dx.doi.org/10.1128\%2FJB.00059-18>; 
BHAGYASREE J.B. et al.Quantum mechanical and spectroscopic (FT-IR, FT-Raman, 1H NMR and UV) investigations of 5-nitro-2-phenylbenzoxazole. Journal of Molecular Structure. Vol. 1063, p. 16-29, 2014;

BHATT, Nikhil. et al.Decolorization of diazo-dye Reactive Blue 172 by Pseudomonas aeruginosa NBAR12. Journal of Basic Microbiology. Vol. 45, n. 6, p. 407-418, 2005;

BHOSALE, Manohar A; BHANAGE, Bhalchandra M. Rapid synthesis of nickel oxide nanorods and its applications in catalysis. Advanced Powder Technology. Vol. 26, p. 422-427, 2015;

BRAY J.; KING E.J.The phosphatase reaction as na AID to identification as an aid to the identification of microorganisms using phenolphthalein phosphate as substrate. The Journal of Pathology and Bacteriology. Vol. 55, p. 305-320, 1943;

CELLIER, Marie. et al. 2-Arylbenzothiazole, benzoxazole and benzimidazole derivatives as fluorogenic substrates for the detection of nitroreductase and aminopeptidase activity in clinically important bacteria.Bioorganic and Medicinal Chemistry. Vol. 19, p. 2903-2910, 2011;

CELLIER, Marie. et al. Novel chromogenic aminopeptidase substrates for the detection and identification of clinically important microorganisms. Bioorganic and Medicinal Chemistry. Vol. 22, p. 5249-5269, 2014;

CLANCY, Cornelius J., NGUYEN, Minh-Hong H. Emergence of Candida auris: An International Call to Arms. Clinical Infectious Diseases. Vol.64, n. 2, p. 141-143, 2017;

COATES, John, Introduction to Infrared Spectrum, A Practical Approach. Encyclopedia of Analytical Chemistry: John Wiley and Sons Ltd. Chichester, 2000;

DEIVASIGAMANI, Charumathi; DAS, Nilanjana. Biodegradation of Basic Violet 3 by Candida krusei isolated from textile wastewat. Biodegradation. Vol. 22, p. 1169-1180, 2011;

DIXIT S.; GARG S. Development of an efficient recombinant bacterium and its application in the degradation of environmentally hazardous azo dyes.International Journal of Environmental Science and Technology. 2018 <https://doi.org/10.1007/s13762-018-2054-7>

ELDIPINA, Elena N. et I. Analytical Biochemistry Direct detection of cysteine peptidases for MALDI-TOF MS analysis using fluorogenic substrates.Analytical Biochemistry. Vol. 567, p. 45-50, 2019.

ELFARASH, Ameer. et al. Azoreductase kinetics and gene expression in the synthetic dyesdegrading Pseudomonas. Bioorganic and Medicinal Chemistry. Vol. 19, n. 9, p. 2903-2910, 2011;

ESLAMI, Hadi. et al. Decolorization and biodegradation of reactive Red 198 Azo dye by a new Enterococcus faecalis-Klebsiella variicola bacterial consortium isolated from textile wastewater sludge. World Journal of Microbiology and Biotechnology. 35:38, 2019 < https://doi.org/10.1007/s11274-019-2608-y >

ESMAELI, Akbar.; KALANTARI, Mona. Bioremoval of an azo textile dye, Reactive Red 198, by Rhizopus oryzae. Journal of Destination and Water treatment. Vol. 57, n. 14, 2016;

GRIST, Roger. Fluorogenics: A New Application of an Old Technology. Clinical Microbiology Newsletter. Vol. 9, n. 8., p.57-64, 1987;

HERNÁNDEZ-SOLÍS, Sandra; RUEDA-GORDILLO, Florencio; ROJAS-HERRERA, Rafael A.Actividad de la proteinasa en cepas de Candida albicans aisladas de la cavidad oral de pacientes inmunodeprimidos, con candidiasis oral y sujetos sanos. Revista Iberoamericana de Micología. Vol. 31, n. 2, p. 137-140;

HODGES, Heather et al.Imaging mycobacterial growth and division with a fluorogenic probe. Proceedings of the National Academy of Sciences of the United States of America. Vol. 115, p. 5271-5276, 2018;

HOLLER, Marcelo. G. et al. Synthesis and spectroscopic characterisation of 2-(2'-hydroxyphenyl)benzazole isothiocyanates as new fluorescent probes for proteins. Journal of Photochemistry and Photobiology A: Chemistry. Vol. 149, p. 217-225, 2002; 
IANDOLO, Donata. et al. Enzyme Production by Solid Substrate Fermentation of Pleurotus ostreatus and Trametes versicolor on Tomato Pomace. Applied Biochemistry and Biotechnology. V.163, p. 4051, 2011;

KANANGARAJ J. et al. Biological method for decolourisation of an azo dye: clean technology to reduce pollution load in dye waste water. Clean Techonology Environmental Policy. Vol. 14, p. 565-572, 2012;

KAUR, Avneet et al. Synthesis, biological evaluation and docking study of N-(2-(3,4,5trimethoxybenzyl)benzoxazole-5-yl) benzamide derivatives as selective COX-2 inhibitor and anti-inflammatory agents. Bioorganic Chemistry. Vol. 81, p.191-202, 2018;

KELLNER, R. et al. Analytical Chemistry: A Modern Approach to Analytical Science. Weinheim: Wiley-VCH, 2th Ed. $1181 \mathrm{pp}, 2004$.

LAMBERT, J. B. et al. Introduction to organic spectroscopy. New York: Macmillan Publishing Company. 1987, 454p.

YOU, Le Xing et al. Extracellular electron transfer of Enterobacter cloacae SgZ-5T via bimediators for the biorecovery of palladium as nanorods . Environmental International. Vol.123, p. 1-9, 2019;

ULDIS M.G.; MUTER K.O. Metabolic response of bacteria to elevated concentrations of glyphosate based herbicide. Ecotoxicology and Environmental Safety. Vol. 173, p. 373-380, 2019;

LUCAS, Marco S. et al. Biodegradation of the diazo dye Reactive Black 5 by a wild isolate of Candida oleophila. Enzyme and Microbial Technology. Vol. 39, n. 1, p. 51-55, 2006;

MALMARTEL A.; GHASAROSSIAN C.Bacterial resistance in urinary tract infections in patients with diabetes matched with patients without diabetes. Journal of Diabetes and Its Complications. V. 30, p. 705-709, 2016;

MANAFI, Mohammed. Fluorogenic and Chromogenic Substrates Used in Bacterial Diagnostics. Microbiological Reviews. Vol. 55, N. 3, p. 335-348, 1991;

NAKANISHI K. Infrared absorption spectroscopy - practical. Holden-Day, Inc., San Francisco and Nankodo Company Ltd., Tokyo. 233 p., 1962.

ORTIZ, Bryan. et al. Molecular identification of Candida species from urinary infections in Honduras. Revista Iberoamericana de Micología. Vol. 35, n. 2, p. 73-7, 2018;

OZER T.T.; DURMAZ S.;YULA E..Antifungal susceptibilities of Candida species isolated from urine culture. $J$ Infect Chemotherapy. Vol. 40, n.9, p. 629-632, 2016;

PADALKAR, Vikas S. et al. Synthesis and antimicrobial activity of novel 2-substituted benzimidazole, benzoxazole and benzothiazole derivatives. Arabian Journal of Chemistry. Vol. 9, n. 2, p. S1125-S1130, 2016;

PAVIA, D. L.; PAMPMAN, G. M.; KRIZ, G. S. Introduction to Spectroscopy. 2. ed. Fort Worth: Harcourt Brace College, 1996, 514p.

RAMALINGAN, Chennan. et al. Synthesis and study of antibacterial and antifungal activities of novel 1-[2(benzoxazol-2-yl)ethoxy]- 2,6-diarylpiperidin-4-ones. European Journal of Medicinal Chemistry. Vol.39, p. 527-533, 2004;

RAMBACH, Allan. New plate medium for facilitated differentiation of Salmonella spp. from Proteus spp. and other enteric bacteria. Applied Environmental Microbiology. Vol. 56, n. 1, p. 301-303, 1990

RAMOS A. et al. Risk factors, clinical presentation and prognosis of mixed candidaemia: a population-based surveillance in Spain. Mycoses. Vol. 59, n. 10, p. 636-43, 2016;

REZAEI-SERESHT, Esmail et al. Synthesis and anticancer activity of new azo compounds containing extended ח-conjugated systems. Chemical Papers. Vol. 71, 2017. <https://doi.org/10.1007/s11696-017-0140-9>; 
ROEGES, Noel.P.G. A Guide to the Complete Interpretation of Infrared Spectra of Organic Structures. Journal of Chemical Education. Vol. 72, n. 4, p. A93, 1995;

SARANGI, Ashish K.; MAHAPATRA, Bipin B; SETHY, Sisir K. Synthesis and Characterization of Tetranuclear Metal Complexes with an Octadentate Azodye Ligand. Chemistry Africa. Vol. 1, p. 117-28, 2018;

SHAH, Maulin. et al. Microbial degradation of azo dye by Pseudomonas spp. MPS-2 by an application of sequential microaerophilic \& aerobic process. American Journal Microbiological Research. Vol. 4, n. 1, p. 105-112, 2013;

SHANG, Yuting. et al. A water-soluble, small molecular fluorescence probe based on 2-(2'-hydroxyphenyl) benzoxazole for $\mathrm{Zn}^{2+}$ in plants. Tetrahedron Letters. Vol. 59, n.45, p. 4003-4007, 2018;

MARY Y.S. et al. FT-IR, FT-Raman, SERS and computational study of 5-ethylsulphonyl-2-(0chlorobenzyl)benzoxazole. Spectrochimica Acta Part A: Molecular and Biomolecular Spectroscopy. Vol. 96, p 617625, 2012;

SILVERSTEIN, R. M.. Identificação espectrométrica de compostos orgânicos. 7. ed. São Paulo: LTC, 2006;

TAN, Liang. et al. Aerobic decolorization and degradation of azo dyes by growing cells of a newly isolated yeast Candida tropicalis TL-F1. Bioresource Technology.Vol. 138, p. 307-313, 2013;

Wiley, New York, 1994;

XUE-MIN DAl et al. Preparation and Properties of High-performance Polyimide Copolymer Fibers Derived from 5Amino-2-(2-hydroxy-5-aminobenzene)-benzoxazole. Chinese Journal of Polymer Science. 2019. <https://doi.org/10.1007/s10118-019-2205-4>

OSTROSKY-ZEICHNER, Luís. Editorial Commentary: Candida glabrata and FKS Mutations: Witnessing the Emergence of the True Multidrug-Resistant Candida. Clinical Infectious Diseases, Vol. 56, n. 12, p. 1733-1734, 2013;

ZEYREK, Celal T. et al. Spectroscopic, quantum mechanical and molecular docking studies of a new benzoxazole compound with an oxidoreductase enzyme and DNA. Journal of Molecular Structure. Vol. 1136, p. 112-126, 2017; 